\title{
Post-traumatic stress disorder
}

\author{
Jonathan I Bisson ${ }^{1}$
}

Cardiff University, University Hospital of Wales, Cardiff CF14 4XW

\section{Correspondence to:} bissonji@cf.ac.uk

BMJ 2007;334:789-93 doi: $10.1136 /$ bmj.39162.538553.80
Vivid descriptions of reactions to traumatic events span many centuries, although their nature has changed over time. ${ }^{1}$ Post-traumatic stress disorder was first recognised as a diagnosable psychiatric disorder in the third edition of the Diagnostic and Statistical Manual of Mental Disorders (DSM-III) ${ }^{\mathrm{w1}}$ and ICD-10 (International Classification of Diseases, 10th edition). ${ }^{2}$ Its very existence continues to attract debate, with several authors arguing that culturally determined, understandable emotions to traumatic events are being pathologised. ${ }^{\mathrm{w} 2}$ However, even its most ardent critics are more concerned by overdiagnosis than by whether post-traumatic stress disorder should be used as a label for the severe mental health problems some people experience after traumatic events. ${ }^{3}$ This article provides an overview of our current understanding of the disorder, who it affects, and the best approaches to its prevention and management.

\section{Methods}

I consulted recent systematic searches used to prepare Cochrane reviews and BMJ Clinical Evidence on prevention and treatment of post-traumatic stress disorder, as well as those used to prepare the National Institute for Health and Clinical Excellence (NICE) guidelines for post-traumatic stress disorder. I searched PubMed using the terms "epidemiology", "neurobiology", and "neuroimaging" in conjunction with "post-traumatic stress disorder". I also used a personal archive of references.

\section{What is post-traumatic stress disorder?}

Box 1 shows the characteristic features of the disorder. It occurs after a traumatic event that involved actual or threatened death or serious injury, or a threat to the physical integrity of self or others. ${ }^{4}$ For the DSM classification, the person must also have experienced intense fear, helplessness, or horror when the event occurred. The symptoms must have been present for at least one month (the one month criterion does not apply in the ICD-10 classification) and, crucially, they must also cause clinically significant distress or impairment in social, occupational, or other important areas of functioning. Acute post-traumatic stress disorder becomes chronic if it lasts for longer than three months. Symptoms usually begin shortly after the trauma, but they are said to have delayed onset if they start at least six months later. Most people diagnosed with delayed onset post-traumatic stress disorder actually had symptoms within six months of the trauma, but they presented late or their symptoms were not recognised initially. For a few patients the onset of symptoms truly is delayed. ${ }^{\mathrm{w} 3}$

\section{Psychological models of the disorder}

Traumatic experiences can usually be assimilated without the development of a pathological response. ${ }^{\mathrm{w} 4}$ If this is unsuccessful, post-traumatic stress disorder can develop, with pathological fear structures characterised by excessive response elements such as avoidance, physiological reactivity, and resistance to modification. ${ }^{\mathrm{w} 5}$ Central to cognitive theories of post-traumatic stress disorder are pre-existing beliefs and models of the world, and the difficulty of assimilating information provided by a traumatic experience into them. ${ }^{56}$ One prominent theory ${ }^{5}$ distinguishes between memories that are easily verbally recalled and give rise to emotions related to the trauma and memories that cannot be deliberately accessed and give rise to symptoms such as dreams and flashbacks. Another theory suggests that the disorder develops when the traumatic

Box 1 | Characteristic symptoms of post-traumatic stress disorder adapted from DSM-IV

Re-experiencing phenomena (at least one required)

- Recurrent and intrusive distressing recollections

- Recurrent distressing dreams

- Acting or feeling as if the events are recurring

- Intense psychological distress to cues

- Physiological reactivity to cues

Avoidance and numbing (at least three required)

- Avoidance of thoughts, feelings, and conversations

- Avoidance of reminders

- Psychogenic amnesia

- Greatly reduced interest in related activities

- Detachment or estrangement feelings

- Restricted range of affect

- Sense of a foreshortened future

Increased arousal (at least two required)

- Difficulty sleeping

- Irritability or outbursts of anger

- Difficulty concentrating

- Hypervigilance

- Exaggerated startle response 
memory induces a sense of current threat promoted by excessively negative appraisals of what happened. ${ }^{6}$

\section{Biological models of the disorder}

Although still in its infancy, with many findings still needing confirmation, our knowledge of the neurobiology of post-traumatic stress disorder is improving. The amygdala receives information about external stimuli and determines their importance. This then triggers emotional responses including a "fight, flight, or freezing" response and alterations in stress hormones and catecholamines. The hippocampus and medial prefrontal cortex (fig 1) influence the response of the amygdala in determining the final fear response. Hippocampal lesions have been associated with a stronger fear response and smaller hippocampal volume has been associated with post-traumatic stress disorder, ${ }^{7}$ although whether this is a cause or an effect of the disorder is unknown. ${ }^{\text {w6 }}$ Neuroimaging studies have shown decreased activity in medial prefrontal and anterior cingulate areas to be correlated with increased activity in the amygdala. ${ }^{8}$ It has therefore been suggested that post-traumatic stress disorder represents a failure of medial prefrontal and anterior cingulate networks to regulate the activity of the amygdala, which results in hyper-reactivity to threat. ${ }^{9}$

One of the most enduring neurophysiological theories has been that of enhanced negative feedback in the hypothalamic-pituitary-adrenal axis. Several studies have found low cortisol concentrations in people with post-traumatic stress disorder and an opposite response to the dexamethasone suppression test than that seen with severe depression. However, this finding has not consistently been supported by

Box 2 | Factors associated with post-traumatic stress disorder ${ }^{1516}$

\section{Pretraumatic factors}

- Previous psychiatric disorder

- Sex (more prevalent in female patients than in male patients)

- Personality (external locus of control greater than internal locus of control)

- Lower socioeconomic status

- Lack of education

- Race (minority status)

- Previous trauma

- Family history of psychiatric disorders

Peritraumatic factors

- Severity of trauma

- Perceived threat to life

- Peritraumatic emotions

- Peritraumatic dissociation

Post-traumatic factors

- Perceived lack of social support

- Subsequent life stress

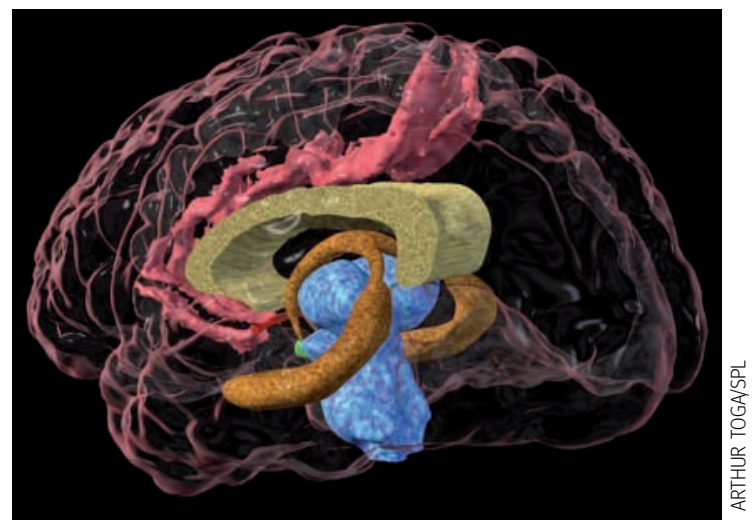

Fig 1 Three dimensional magnetic resonance imaging scan showing the hippocampus (mid-brown)

more recent studies. ${ }^{10}$ The increased catecholamine concentrations in people with the disorder have also caused much interest. It has been suggested that an initial adrenergic surge may be associated with the consolidation of traumatic memories. ${ }^{11}$

\section{Who is affected?}

The recent replication of the US national comorbidity survey of 5692 adults found that the lifetime prevalence of post-traumatic stress disorder was $6.8 \%{ }^{12}$ and the 12 month prevalence was $3.5 \%{ }^{13}$; about a third of those affected had a severe form of the condition. However, similar epidemiological studies of adolescents and young adults in Germany ${ }^{\mathrm{w} 7}$ and adults in Australia $^{\mathrm{w} 8}$ produced far more conservative estimates - prevalence at 12 months was $0.7 \%$ and $1.2 \%$, respectively. Comorbidity rates are often more than $80 \%{ }^{14}$ The most common comorbid conditions are depressive disorders, panic disorder, other anxiety disorders, and substance misuse or dependence. Anyone can develop the disorder after a traumatic event, but the incidence increases with the severity of the trauma. Studies have reported an incidence of more than 50\% for rape, ${ }^{14}$ w9 $30-40 \%{ }^{\mathrm{w} 10}$ for disasters, and around $19 \%$ for veterans of the Vietnam war. ${ }^{\text {w1 }}$ Rates for other traumas including accidents and non-physical assaults tend to be lower. ${ }^{14}{ }^{\text {w9 }}$ Factors associated most closely with the development of post-traumatic stress disorder are perceived lack of social support and peritraumatic dissociation, but neither increase the risk by more than $50 \% .{ }^{1516}$ Box 2 lists the factors associated with the disorder across several studies.

\section{Immediate reactions after trauma}

Many people experience traumatic stress symptoms shortly after traumatic events. More than $90 \%$ of female victims of sexual assault satisfy the symptom criteria for post-traumatic stress disorder within a week of the event, ${ }^{\text {w12 }}$ and $31 \%$ of 1010 Londoners described substantial stress 11-13 days after the bombings in London on 7 July 2005. ${ }^{17}$ Prospective research suggests that rates reduce rapidly over time. After the 11 September terrorist attacks in New York, probable post-traumatic stress disorder in people living south of 
Box 3 Items on trauma screening questionnaire ${ }^{w 13}$

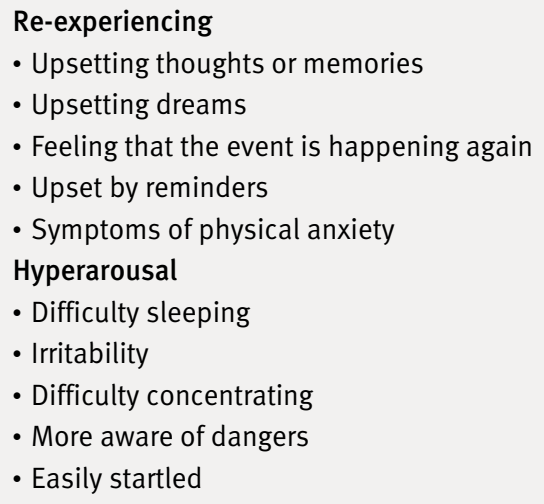

110th Street reduced from $7.5 \%$ at one month to $1.6 \%$ at four months and $0.6 \%$ at six months. ${ }^{18}$ This recovery trajectory is important when deciding how best to provide for individuals after traumatic events.

\section{Detecting the disorder}

Despite the associated factors it is difficult to predict exactly who will develop post-traumatic stress disorder after a traumatic event. Various screening instruments have been developed, ${ }^{19}$ and one of the simplest - the 10 item trauma screening questionnaire ${ }^{\text {w13 }}$ (box 3) - is one of the best validated. ${ }^{19}$ This questionnaire is probably more helpful in detecting people with the chronic disorder than as a predictive screening instrument shortly after a traumatic event. This is because it produces a high number of false positives unless a high incidence of the chronic disorder is expected. ${ }^{\mathrm{w} 14} \mathrm{~A}$ full assessment that equates to a standard mental health assessment is needed to diagnose the disorder. This should look at presentation, background, mental state examination result, risk assessment result, social factors including support network, and information from other sources including relatives.

\section{Preventing the disorder}

Formal psychological interventions targeted at everyone involved in traumatic events have been ineffective. $^{20 \text { w15 w16 }}$ Meta-analyses have indicated no differences between people who received an intervention and those who did not in the first six months. ${ }^{20 \text { w15 } 16}$ Some studies have reported more negative outcomes in people who receive one-off individual interventions based on critical incident stress debriefing, particularly those with higher levels of symptoms. ${ }^{\text {w17-w19 }}$ This has led to concerns about this form of input and recommendations that it should not be used. ${ }^{20}$ More attention is now being focused on developing stepped or stratified models of response in which immediate practical, social, and emotional support is offered by non-mental health professionals. People with severe ongoing symptoms are offered a formal assessment and a more complex intervention if post-traumatic stress disorder that is not improving is detected..$^{2021}$
The most researched complex early interventions have used trauma focused cognitive behaviour therapy over four to 12 sessions, starting from one to three months after the traumatic event. ${ }^{\text {w20-w22 }}$ These contain components similar to those used to treat the chronic disorder (box 4). No research has suggested a formal role for the early prescription of drugs. However, guidelines from the United Kingdom's National Institute for Health and Clinical Excellence suggest that acute phase symptoms, such as severe insomnia, could be managed with hypnotics or antidepressants. ${ }^{20}$ Concern about prescribing of benzodiazepines has resulted in many prescribers preferring to recommend a sedative antidepressant or an $\alpha$ agonist, such as prazosin. ${ }^{\text {w23 }}$

\section{Managing chronic post-traumatic stress disorder}

A wide range of psychological and pharmacological approaches have been used to treat the chronic disorder. Unfortunately, no good direct comparisons of the two approaches have been carried out, making it difficult to determine their relative efficacies.

\section{Psychological treatment}

The psychological treatments with the best evidence are trauma focused cognitive behaviour therapy, which comprises varying combinations of exposure therapy and trauma focused cognitive therapy, and eye movement desensitisation and reprocessing. ${ }^{2022} 23$ Both are individual treatments usually provided over the course of up to 12 sessions (box 4; fig 2). Exposure therapy commonly involves the patient describing the traumatic event in great detail, taping this account, and listening to it repeatedly. In trauma focused cognitive therapy, distorted beliefs and misinterpretations about the traumatic event and its consequences are considered and challenged. In eye movement desensitisation and reprocessing, the recipient focuses on the traumatic experience, associated thoughts, emotions, and

Box 4 | Trauma focused psychological treatments

Exposure therapy

- Repeated confrontation of traumatic memories, often through detailed recounting of the traumatic experience

- Repeated exposure to avoided situations and those that elicit fear that are now safe but which are associated with the trauma

Trauma focused cognitive therapy

- Modification of misinterpretations that lead to overestimation of current threat

- Modification of other beliefs related to the traumatic experience and the individual's behaviour during the trauma (for example, guilt and shame)

Eye movement desensitisation and reprocessing

- Standardised procedure using bilateral physical stimulation (eye movements, taps, or tones) while the patient focuses on memories and associations

- This form of treatment is thought to stimulate information processing to help produce an adaptive contextualised memory 


\section{Unanswered questions}

- Are the changes in brain structure and function found in post-traumatic stress disorder a result of the disorder?

-What is the most appropriate way to respond psychosocially after a traumatic event?

- Are trauma focused psychological treatments more effective than drug treatments?

- Can the response to treatment be predicted?

-What are the most effective treatments for children and adolescents?

sensations while receiving bilateral physical stimulation, most commonly by following the therapist's finger as it is moved from side to side.

Non-trauma focused stress management has a positive but less pronounced effect. Group treatment seems to be less effective than individual treatment. A recent metaanalysis found no significant benefits over waiting list controls for non-trauma focused treatments such as supportive therapy, non-directive counselling, psychodynamic therapies, and hypnotherapy. ${ }^{20}$ Using a predetermined threshold, only trauma focused cognitive behaviour therapy and eye movement desensitisation and reprocessing produced significant clinical improvements, and no major differences were found between the two in head to head comparison studies. ${ }^{20}$ w24 w25

\section{Drug treatment}

The efficacy of various drugs has provoked considerable debate and conflicting recommendations. A Cochrane review ${ }^{24}$ and the American Psychiatric Association ${ }^{25}$ reported benefits for selective serotonin reuptake inhibitors - sertraline and paroxetine. NICE do not recommend sertraline, mainly because data from unpublished studies were included in their meta-analysis, and no drug satisfied their predetermined threshold for significant clinical improvement. ${ }^{20}$ Paroxetine, mirtazapine, amitryptyline, and phenelzine were all significantly better than placebo, although studies of all but paroxetine had small sample sizes. Adding olanzapine to an antidepressant also seemed to be better than adding placebo to augment treatment in people with chronic post-traumatic stress disorder who did not respond fully to antidepressant drugs alone.

\section{Management guidelines for the chronic disorder}

The NICE management guidelines for the chronic disorder recommend that all patients should be

\section{Tips for general practitioners}

- Consider asking questions about re-experiencing traumatic events or hyperarousal to detect post-traumatic stress disorder

- Reactions to traumatic events usually resolve without treatment but can become problematic

- If symptoms are not resolving one to three months after the traumatic event consider trauma focused cognitive behaviour therapy

- Trauma focused psychological treatment is the treatment of choice for chronic post-traumatic stress disorder

- Drug treatment should be considered as second line treatment

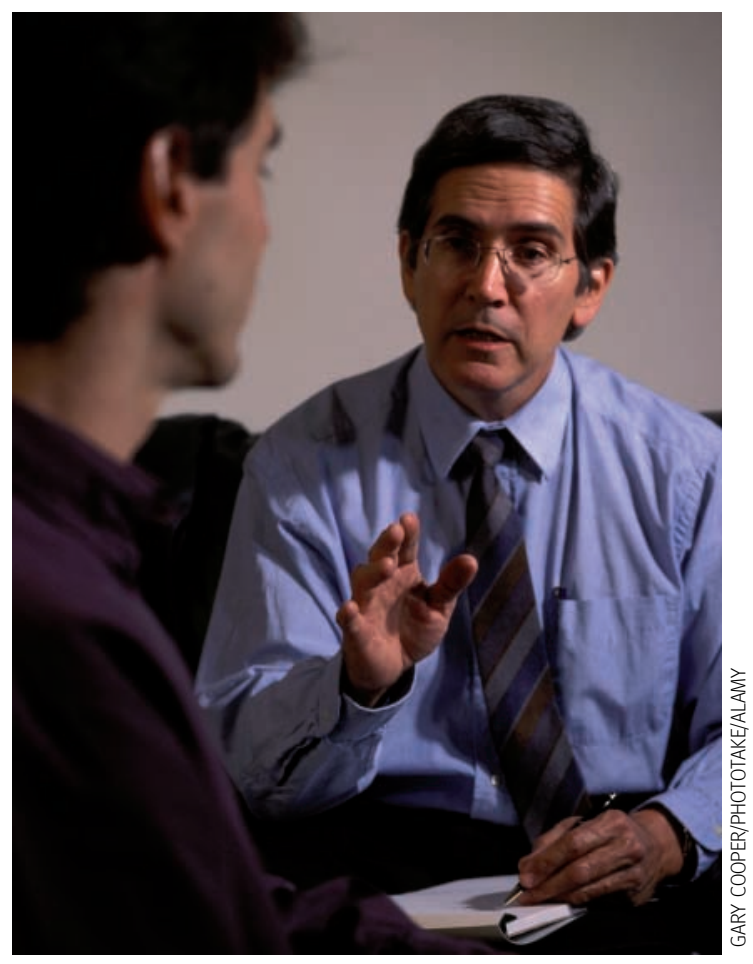

Fig 2 | Trauma focused psychological treatment

offered a course of trauma focused cognitive behaviour therapy or eye movement desensitisation and reprocessing, normally on an individual outpatient basis. ${ }^{20}$ Trauma focused psychological treatment should usually be given for eight to 12 sessions, with some sessions lasting for 90 minutes if the trauma is considered during the session. The number of sessions may need to be increased, especially after multiple traumatic events and when accompanied by comorbidity or traumatic bereavement. An alternative trauma focused treatment or augmentation with drug treatment should be considered if no improvement is seen. Paroxetine and mirtazapine are recommended for general use, and amitriptyline and phenelzine under specialist supervision. Other factors may precipitate prescription of drugs including patient choice, serious ongoing threat, and lack of immediate availability of psychological treatment. Caution is needed when prescribing because of the well documented problems associated with some antidepressants in recent years. ${ }^{26} 6$

\section{Prognosis}

Few longitudinal follow-up studies of post-traumatic stress disorder have been carried out. In one of the largest cross sectional studies, more than a third of people reported having the disorder six years after they first developed it, with a 50\% chance of remission at two years. ${ }^{14}$ However, these results are of limited value in determining prognosis accurately. Whatever the true figure, for many people with the chronic disorder their condition is severe and enduring. ${ }^{\text {w27 }}$ Treatment can help some people many years after they developed the 


\section{SUMMARY POINTS}

Post-traumatic stress disorder is a major cause of distress and reduced functioning after traumatic events in people of all ages

No routine intervention has been shown to prevent this disorder

Trauma focused cognitive behaviour therapy is the treatment of choice within three months of a traumatic event

Trauma focused cognitive behaviour therapy or eye movement desensitisation and reprocessing are the treatments of choice for chronic post-traumatic stress disorder Drug treatment is a second line treatment for the disorder

disorder, so that the duration of symptoms should not influence the decision about whether or not to offer treatment.

\section{Children and adolescents}

This article has focused on adults, but children and adolescents often experience similar symptoms after traumatic events. Younger children often present with other phenomena directly related to the trauma (such as repetitive play and drawing) or more general emotional and behavioural difficulties such as regression, new fears, and aggression..$^{20}$ Research on post-traumatic stress disorder in children and adolescents lags behind research in adults, but it is increasing. Some evidence suggests that trauma focused cognitive behaviour therapy adapted for age, circumstances, and level of development should be offered to children and adolescents with the disorder, irrespective of the traumatic event. ${ }^{20}$ The NICE guidelines recommend family involvement

\section{Additional educational resources}

\section{Resources for healthcare professionals}

- NICE (www.nice.org.uk/page.aspx?o=CG026fullguideline)—Full NICE guidelines for post-traumatic stress disorder

- NICE (www.nice.org.uk/page.aspx?o=CG026quickrefguide)—Quick reference guide to NICE guidelines for post-traumatic stress disorder

- European Society for Traumatic Stress Studies (www.estss.org)-Website of European network for professionals in the field of traumatic stress

- International Society for Traumatic Stress Studies (www.istss.org)-Forum for the sharing of research, clinical strategies, public policy concerns, and theoretical formulations on trauma

- National Center for Post-traumatic Stress Disorder (http://www.ncptsd.va.gov/)— Educational resource concerning consequences of traumatic stress, for a variety of audiences

- David Baldwin's trauma information pages (www.trauma-pages.com/)-Site providing information for clinicians and researchers in the traumatic stress field

\section{Information resources for patients}

- NICE (www.nice.org.uk/page.aspx?o=CG026publicinfo)—Public information on the NICE guidelines for post-traumatic stress disorder

- Post-traumatic stress disorder (www.ptsd.org.uk/)—Website created for ex-service men and women but also used by other people

- National Institute for Mental Health. Post-traumatic stress disorder (www.nimh.nih. gov/healthinformation/ptsdmenu.cfm)—Public information on PTSD.

- Royal College of Psychiatrists. Post-traumatic stress disorder (www.rcpsych.ac.uk/ mentalhealthinformation/mentalhealthproblems/posttraumaticstressdisorder/ posttraumaticstressdisorder.aspx)—Leaflet on post-traumatic stress disorder

- Herbert C, Wetmore A. Overcoming traumatic stress. London: Constable and Robinson, 1999. where appropriate, and they conclude that there is currently no good evidence for widely used treatments such as play therapy, art therapy, or family therapy for posttraumatic stress disorder. ${ }^{20}$

Competing interests: None declared.

1 Jones E, Wessely S. Shell shock to PTSD: military psychiatry from 1900 to the Gulf War. Maudsley monograph 47. Hove: Psychology Press, 2005.

2 WHO. The ICD-10 classification of mental and behavioural disorders: clinical descriptions and diagnostic guidelines. Geneva: WHO, 1992.

3 Summerfield $D$. The invention of post-traumatic stress disorder and the social usefulness of a psychiatric category. BMJ 2001;322:95-8

4 American Psychiatric Association. Diagnostic and statistical manual of mental disorders (DSM-IV). 4th ed. Washington, DC: American Psychiatric Association, 1994.

5 Brewin C, Dalgleish T, Joseph S. A dual representation theory of posttraumatic stress disorder. Psychol Rev 1996;4:670-86.

6 Ehlers A, Clark DM. A cognitive model of posttraumatic stress disorder. Behav Res Ther 2000;38:319-45.

7 Bremner JD, Staib LH, Kaloupek D, Southwick SM, Soufer R, Charney DS. Neural correlates of exposure to traumatic pictures and sound in Vietnam combat veterans with and without posttraumatic stress disorder: a positron emission tomography study. Biol Psychiatry 1999;45:806-16.

8 Shin LM, Orr SP, Carson MA, Rauch SL, Macklin ML, Lasko NB, et al. Regional cerebral blood flow in the amygdala and medial prefrontal cortex during traumatic imagery in male and female Vietnam veterans with PTSD. Arch Gen Psychiatry 2004;61:168-76.

9 Shin LM, Rauch SL, Pitman RK. Amygdala, medial prefrontal cortex, and hippocampal function in PTSD. Ann New York Acad Sci 2006;1071:67-79.

10 Young EA, Breslau N. Cortisol and catecholamines in posttraumatic stress disorder: an epidemiologic community study. Arch Gen Psychiatry 2005;61:394-401.

11 Pitman RK, Altman B, Greenwald E, Longpre RE, Macklin ML, Poire RE, et al. Psychiatric complications during flooding therapy for posttraumatic stress disorder. J Clin Psychiatry 1991;52:17-20.

12 Kessler RC, Chiu WT, Demler O, Walters EE. Prevalence, severity and comorbidity of 12-month DSM-IV disorders in the national comorbidity survey replication. Am Med Assoc 2005;62:617-27.

13 Kessler RC, Berglund P, Demler O, Jin R, Merikangas KR, Walters EE. Lifetime prevalence and age-of-onset distributions of DSM-IV disorders in the national comorbidity survey replication. Am Med Assoc 2005;62:593-602.

14 Kessler RC, Sonnega A, Bromet E, Hughes M, Nelson CB. Posttraumatic stress disorder in the national comorbidity survey. Arch Gen Psychiatry 1995;52:1048-60.

15 Brewin CR, Andrews B, Valentine JD. Meta-analysis of risk factors for posttraumatic stress disorder in trauma-exposed adults. J Consult Clin Psychol 2000;68:748-66.

16 Ozer EJ, Best SR, Lipsey TL, Weiss, DS. Predictors of post-traumatic stress disorder and symptoms in adults: a meta-analysis. Psychol Bull 2003;129:52-73.

17 Rubin GJ, Brewin C, Greenberg N, Simpson J, Wessely S. Psychological and behavioural reactions to the bombings in London on 7 July 2005: cross sectional survey of a representative sample of Londoners. BMJ 2005;331:606-11.

18 Galea S, Vlahov D, Resnick H, Ahern J, Susser E, Gold J, et al. Trends of probable post-traumatic stress disorder in New York City after the September 11 terrorist attacks. Am J Epidemiol 2003;158:514-24.

19 Brewin CR. Systematic review of screening instruments for adults at risk of PTSD. / Trauma Stress 2005;18:53-62.

20 National Collaborating Centre for Mental Health. Post-traumatic stress disorder: the management of PTSD in adults and children in primary and secondary care. London/Leicester: Gaskell and BPS, 2005.

21 Bisson Jl, Roberts N, Macho G. The Cardiff traumatic stress initiative: an evidence-based approach to early psychological intervention following traumatic events. Psychiatr Bull 27:145-7.

22 Bradley R, Greene J, Russ E, Dutra L, Westen D. A multidimensional meta-analysis of psychotherapy for PTSD. Am J Psychiatry 2005;162:214-27.

23 Bisson J, Andrew M. Psychological treatment of post-traumatic stress disorder (PTSD) [Cochrane review]. Cochrane Database Syst Rev 2005;(2):CD003388.

24 Stein DJ, Ipser JC, Seedat S. Pharmacotherapy for post traumatic stress disorder (PTSD) [Cochrane review]. Cochrane Database Syst Rev 2006;(1):CD002795.

25 American Psychiatric Association. Practice guideline for the treatment of acute stress disorder and post traumatic stress disorder. Washington: APA, 2004. 